\title{
Analyst
}

MINIREVIEW

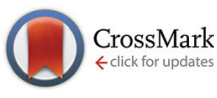

Cite this: Analyst, 2015, 140, 4325

\section{Label-free monitoring of plasmonic catalysis on the nanoscale}

Received 31st March 2015 Accepted 6th May 2015

DOI: 10.1039/c5an00630a www.rsc.org/analyst

\author{
Zhenglong Zhang, ${ }^{a}$ Tanja Deckert-Gaudig ${ }^{a}$ and Volker Deckert*a,b
}

Plasmonics is the description of specific light matter interactions of metallic structures. In general the size of such structures is well in the nanometer regime and also determines such specific characteristics as color, field confinement etc. Plasmon-induced hot electrons play a vital role in so-called plasmonic catalysis, a field that has recently attracted attention as a new reaction platform. Current reports introduce such nanoscale catalysis as an effective approach to concentrate the energy of visible light and direct it to adsorbed molecules, thereby increasing the chemical reaction rate, and controlling the reaction selectivity. In this review, we present various plasmon-catalyzed reactions specifically monitored with Raman spectroscopy, namely surface-enhanced Raman scattering (SERS), remote SERS (Re-SERS) and tip-enhanced Raman scattering (TERS). These techniques utilize the signal enhancing effect of the metal nanoparticles. However, at the same time they can be used to control the actual reactivity. In the first part, the mechanism of plasmonic catalysis is introduced. Then it is shown how catalytic reactions can be spectroscopically investigated far beyond the diffraction limit using TERS. Finally, the sensitivity of the methods is discussed.

\section{Introduction}

In the presence of catalysts the activation barrier that has to be overcome to start a chemical reaction decreases and the reaction can be carried out under milder conditions. At the same

${ }^{a}$ Leibniz Institute of Photonic Technology - IPHT, Albert-Einstein-Str. 9, 07745 Jena, Germany. E-mail: volker.deckert@ipht-jena.de

${ }^{b}$ Institute of Physical Chemistry and Abbe Center of Photonics, Friedrich-Schiller University Jena, Helmholtzweg 4, 07743 Jena, Germany time the reaction rate and efficiency can increase. The catalyst is not consumed during the reaction and can often be recycled. ${ }^{1-3}$ In this field photocatalysis is an emerging and economically viable technology, which can be initiated by the inexhaustibly abundant, clean and safe energy from the sun. ${ }^{4}$ An example of high public interest is the production of the clean fuel $\mathrm{H}_{2}$ that is obtained from the splitting of water by solar photocatalysis in the presence of $\mathrm{TiO}_{2}{ }^{5}$

Recently, surface plasmons (SPs) excited by visible light on metal nanostructures have been used to initiate chemical reac-



Zhenglong Zhang
Zhenglong Zhang studied physics at Shaanxi Normal University and Institute of Physics, Chinese Academy of Sciences, China, and received his Ph.D. in optics in 2013. Then he worked as a Postdoc and AvH Fellow in Prof. V. Deckert's Group at Leibniz Institute of Photonic Technology, Germany. His research interests are in tipenhanced Raman spectroscopy (TERS) and plasmonic catalysis.

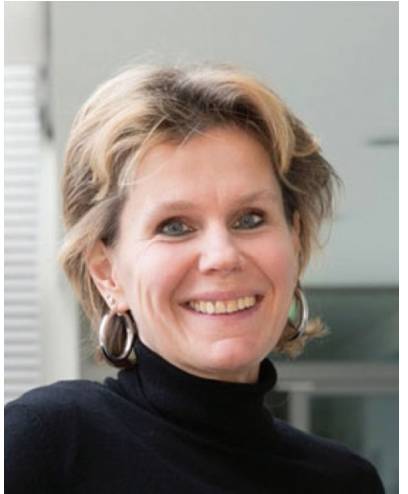

Tanja Deckert-Gaudig
Tanja Deckert-Gaudig studied chemistry at the University of Würzburg and received her Ph.D. in organic chemistry in the group of Prof. S. Hünig in 1997. After her parental leave she switched to physical chemistry in 2002. Since then she has been working on Raman spectroscopy and scanning probe microscopy in the group of Prof. $V$. Deckert. In 2009 she moved to the Leibniz Institute of Photonic Technology (IPHT) in Jena. With tipenhanced Raman spectroscopy (TERS) she studies and chemically characterizes organic and biological surfaces on the nanoscale. Her main focus is set on protein species such as amyloid fibrils. 
tions where the plasmonic nanostructure acts as the catalytic active site. ${ }^{6-8}$ If such a reaction is followed by Raman spectroscopy the plasmonic nanostructure is catalyst and nanoscale signal enhancer, simultaneously. This so-called surface heterogeneous plasmonic catalysis gains significant attention due to its high throughput and low energy requirements as reported in studies on molecular dimerization and dissociation reactions, e.g. dissociation of hydrogen, ${ }^{9,10}$ water, ${ }^{11,12}$ hydrocarbon conversion, ${ }^{13,14}$ etc.

Surface plasmons are collective oscillations of free electrons that exist at the metal-dielectric interface, where the real part of the dielectric function changes sign across the interface. ${ }^{15,16}$ Normally, SPs consist of localized SPs (LSPs) and propagating SPs (PSPs). ${ }^{17}$ LSPs are charge density oscillations confined to the surface of metallic nanoparticles. ${ }^{18}$ LSP resonance (LSPR), the excitation of LSPs by incident light at a special wavelength where resonance occurs, results in a huge enhancement of the local electromagnetic (EM) field. Such EM field enhancements are the underlying physical basis for surface-enhanced Raman scattering (SERS) ${ }^{19}$ and tip-enhanced Raman scattering (TERS). ${ }^{20}$ PSPs are the result of a collective excitation of conduction electrons that propagate in a wave-like manner along the metaldielectric interface, and are confined to the vicinity of the interface. ${ }^{21}$ The remote-excitation measurement, e.g. remote excitation SERS (Re-SERS), ${ }^{22}$ can be explained by PSPs.

In addition to the EM enhancement, LSPs excited on silver or gold nanoparticle surfaces can decay non-radiatively into "hot electrons" with a high energy between the Fermi and vacuum energy level. ${ }^{23-26}$ If hot electrons scatter into an excited state of the absorbed molecules triggering a chemical reaction by reducing the activation energy, this is called a "plasmon catalyzed reaction". A scheme of the proposed mechanism of plasmonic catalysis is shown in Fig. 1. Hot electrons initially generated from the plasmon decay on the silver or gold nanostructures' surface, soon lose coherence and form a

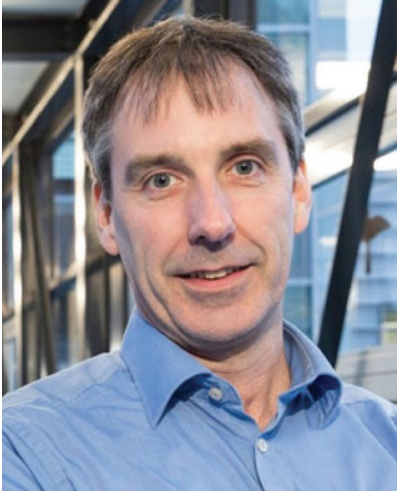

Volker Deckert
Volker Deckert is a Professor at the Institute of Physical Chemistry at the Friedrich-Schiller University, Jena and also heads the Nanoscopy department at the Leibnitz Institute of Photonic Technologies, Jena. He received his Ph.D. from the University of Würzburg, working on Raman spectroscopy. As a post-doc his research focus shifted towards non-linear and time-resolved laser spectroscopy at the University of Tokyo and KAST, in Kawa-

saki. Since his habilitation at the ETH Zurich, he does research on near-field optical spectroscopy, a subject he still pursues and currently applies to biological samples and to the investigation of chemical reactivity on the nanometer scale.

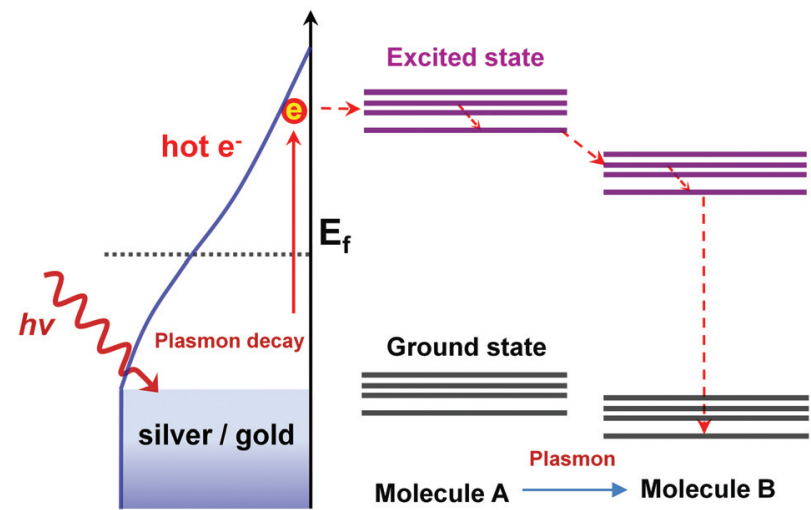

Fig. 1 Sketch of the plasmonic catalysis mechanism. Laser excitation of silver or gold nanostructures, generates hot electrons. Due to a plasmon decay the electrons can be transferred to an excited state of molecule A, from there a catalyzed reaction can be initiated to result in the final product, molecule B. Adapted from ref. 26.

non-equilibrium Fermi-Dirac type distribution. The hot electrons in the high energy level have sufficient energy to transfer to the excited state of molecule A, creating a transient negative ion. This negative ion of molecule A travels to the excited state of molecule B and transfers the electron back to the silver or gold nanostructures' surface, where it returns to the ground state of molecule B and finishes the plasmon catalyzed reaction of molecule A to B.

Based on the same principles of LSPs and PSPs, plasmonic catalysis can be tracked on the nanoscale by SERS and TERS. In this review, we focus on recent advances in the field of plasmon catalysis on the nanoscale. The presented nanoscaletechniques including SERS, remote SERS and TERS used in plasmonic catalysis are reviewed, respectively. First, the early results of plasmon catalyzed reactions observed by SERS and remote-SERS techniques are introduced. The well-studied plasmon catalyzed reaction of $p$-nitrothiophenol ( $\mathrm{pNTP})$ or $p$-aminothiophenol (pATP) molecules to $p, p$-dimercaptoazobenzene (DMAB) are reviewed in detail. Then, it is shown how TERS can be utilized for investigating plasmonic catalysis with high spatial resolution beyond the diffraction limit. The controlled catalytic processes and localized temperature of plasmonic catalysis revealed by TERS are reviewed. Finally, it is demonstrated that plasmonic catalysis can be conducted even at the single molecule level. A new behaviour of pNTP could be monitored on the single molecule level, which strongly differs from previous reports as separated pNTP molecules exclusively react to thiophenol (TP).

\section{Plasmonic catalysis in SERS and Re-SERS}

\subsection{Short introduction of SERS and Re-SERS}

Raman scattering is an inelastic scattering of photons from a molecule in which the frequency change from the excitation 
frequency corresponds to the vibrational energy levels. ${ }^{27}$ Since Raman spectra provide spectroscopic fingerprints of molecules it plays an important role in non-destructive and label-free detection, and biological and chemical sensing. ${ }^{28}$ However, Raman spectroscopy suffers from low sensitivity because of the very low cross section $\left(\sim 10^{-27}-10^{-30} \mathrm{~cm}^{2} \mathrm{sr}^{-1}\right.$ per molecule), which limits the applications of Raman sensing in plasmonic catalysis where often only minute amounts of sample are present. $^{29}$

The phenomenon of LSPR on the nanoscale on metal surfaces led to the SERS that strongly enhances Raman signals of molecules adsorbed on rough metal surfaces. In this way insensitive Raman spectroscopy evolved into a highly sensitive technique. $^{30,31}$ Normally, the enhancement factor of SERS is more than $10^{6}$, depending on the size and shape of the nanostructures, incident light wavelength, distance between molecule and surface, etc. ${ }^{32-34}$ Here, LSPR not only generates the hot electrons necessary for plasmonic catalysis but also enables SERS to monitor the reaction.

As mentioned, PSPs can propagate along a plasmonic waveguide, which allows the transfer of light over micrometres through structures with sub-diffraction limited diameters. ${ }^{35-37}$ Using PSPs as a remote excitation source, a novel technique Re-SERS was first reported in 2009. ${ }^{22,38}$ A schematic comparison of conventional SERS and Re-SERS is shown in Fig. 2. In a SERS experiment, the incident light is focused on the detecting target, which is called "local SERS". In contrast, the ReSERS refers to the focused spot of incident light that is far from the target, which is excited by the PSPs. ${ }^{39}$ Compared with local SERS, Re-SERS can avoid sample damage from direct laser irradiation, improve the signal to noise ratio, and lead to miniaturized photonics and high-resolution microscopy/spectroscopy. Using the Re-SERS technique, the remote investigation provides a novel way to perform sensing measurements of SERS and gives access to systems where traditional SERS is unsuitable, such as remote Raman optical activity, ${ }^{40,41}$ biomolecules in cells detecting, ${ }^{42,43}$ and also plasmonic catalysis. $^{44-46}$

\subsection{Plasmonic catalysis in SERS}

As a typical plasmon catalyzed reaction, pNTP adsorbed on plasmonic nanostructures reacts exclusively to DMAB, which was first reported by Dong et al. in $2011 .{ }^{47}$ The plasmon cata-
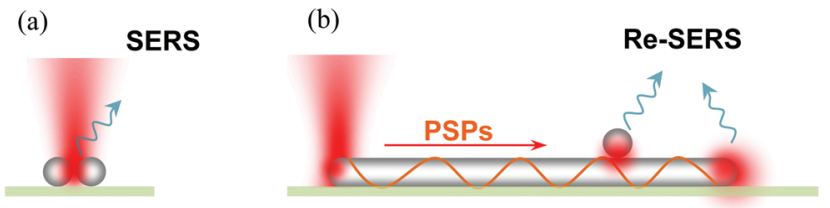

Fig. 2 Sketch of SERS and Re-SERS in an Ag nanoparticle-nanowire system. (a) Conventional SERS, the Raman signal is directly obtained from the nanoparticles. (b) Re-SERS signals are collected at the junction or end of a nanowire induced by the PSPs propagated along the nanowire when excited at the opposite end. lyzed dimerization of pNTP to DMAB is characterized by the disappearance of the Raman band at $1332 \mathrm{~cm}^{-1}\left(\nu_{\mathrm{NO}_{2}}\right)$, and the appearance of new Raman bands around $1140\left(\beta_{\mathrm{C}-\mathrm{H}}\right), 1387$ $\left(\nu_{\mathrm{NN}}+\nu_{\mathrm{CC}}+\nu_{\mathrm{C}-\mathrm{N}}\right)$ and $1432\left(\nu_{\mathrm{NN}}+\nu_{\mathrm{CC}}+\beta_{\mathrm{C}-\mathrm{H}}\right) \mathrm{cm}^{-1}$. This observation can be explained by the reduction of the $\mathrm{NO}_{2}$ group and the formation of the $-\mathrm{N}=\mathrm{N}-$ unit in DMAB. As shown in Fig. 3, with increasing time dimerization of pNTP to DMAB can be confirmed by the afore-mentioned bands in SERS by using silver nanoparticles on $\mathrm{Cu}$ and $\mathrm{Ag}$ films. It was found that this reaction strongly depends on substrate, wavelength and time. So, this plasmonic catalysis can be controlled accordingly. For example, irradiating silver nanoparticles on a copper film with $514 \mathrm{~nm}$ results in a completed reaction within minutes (see Fig. 3a), while on irradiating Ag nanoparticles with $633 \mathrm{~nm}$ on a silver film the reaction takes several hours (see Fig. 3b). The difference is attributed to different plasmon intensities in the gap between $\mathrm{Ag}$ nanoparticles and the $\mathrm{Cu}$ or $\mathrm{Ag}$ film, which can be estimated from the local EM field intensity distribution. pNTP and its amino analogue can be considered as model compounds for plasmon catalysis studies under different experimental conditions and acts a kind of standard to exploit related detection techniques.

In 2010, Fang, Huang and Canpean independently discovered that $p$-aminothiophenol (pATP) can also react to DMAB on SERS active substrates like silver or gold nanoparticles. ${ }^{13,14,48}$ As shown in Fig. 4, the catalyzed reaction of pATP to DMAB was investigated using different substrate dependent experiments, namely SERS, surface mass spectra, and potential dependent SERS. The results agree well with theoretical calculations utilizing density functional theory. These experiments investigate several aspects to fully understand previous (mis-)interpretations of pATP SERS spectra. ${ }^{49-52}$ Until recently, there were debates regarding whether the reac-
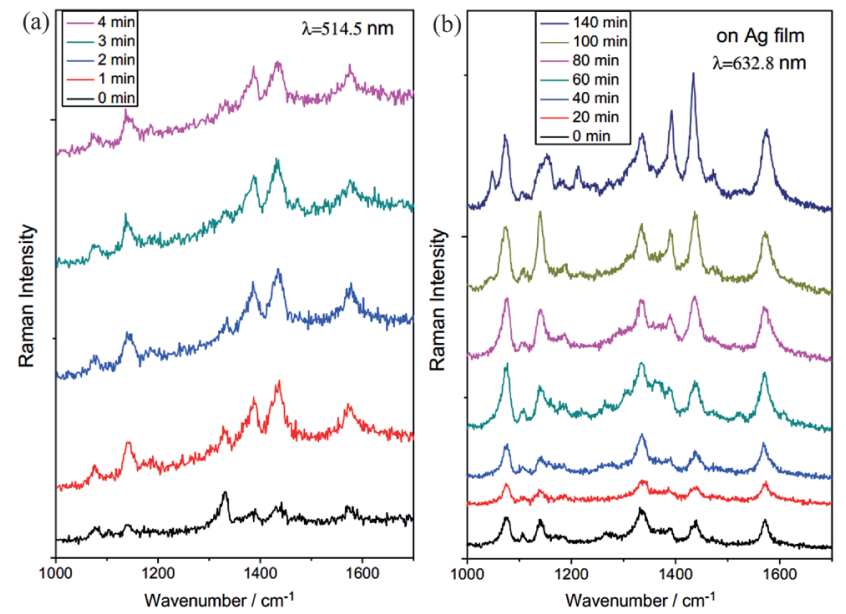

Fig. 3 Time dependent SERS measurement of pNTP adsorbed on Ag nanoparticles on $\mathrm{Cu}$ and Ag films, respectively. (a) On Cu film at $514 \mathrm{~nm}$, and (b) on Ag film at $632.8 \mathrm{~nm}$. The peak at $1332 \mathrm{~cm}^{-1}$ is attributed to the $\mathrm{NO}_{2}$ vibration of pNTP and the peaks at 1140,1387 and $1432 \mathrm{~cm}^{-1}$ are attributed to $\mathrm{N}=\mathrm{N}$ vibrations of $\mathrm{DMAB}$. Adapted from ref. 47 . 


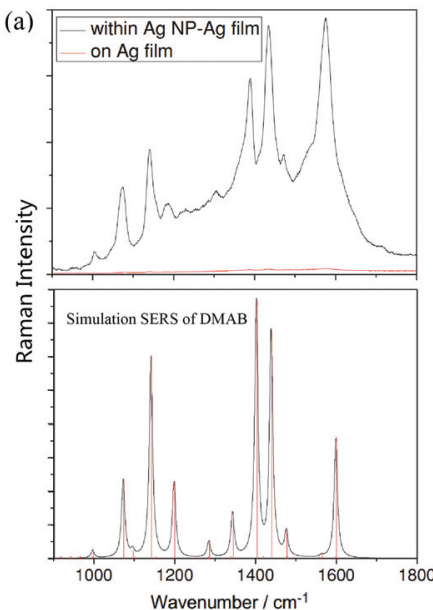

(b)
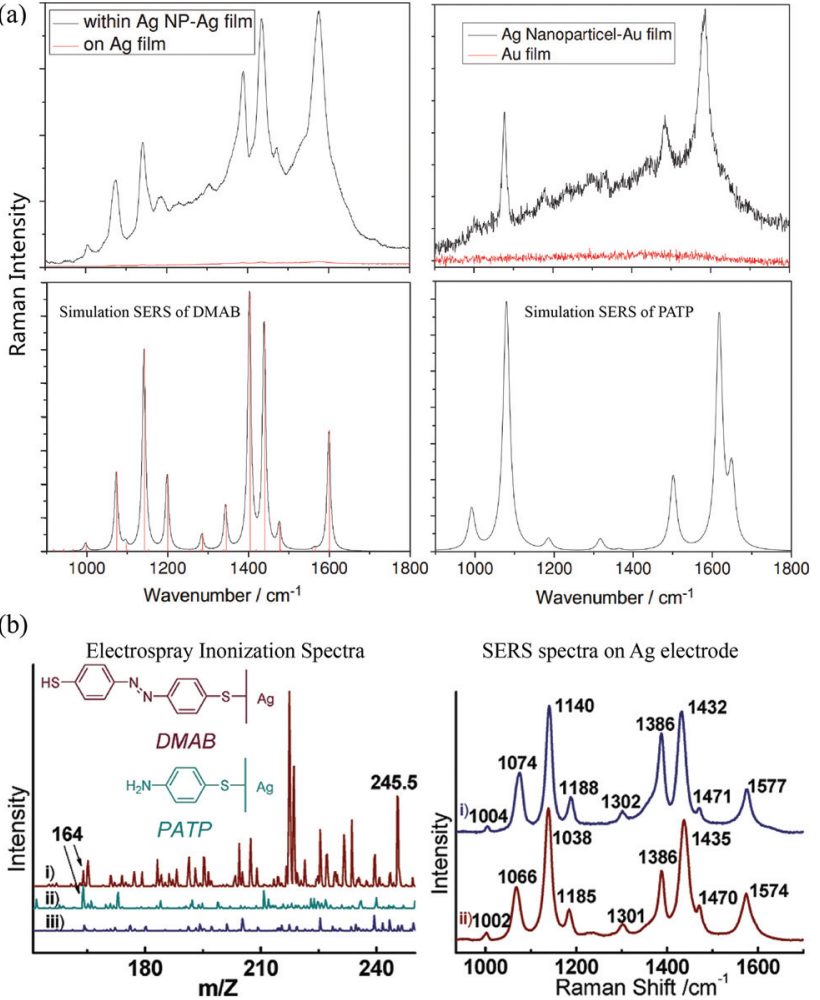

SERS spectra on Ag electrode

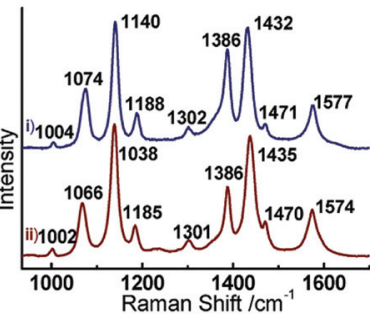

Fig. 4 Reaction of PATP to DMAB confirmed by SERS, surface mass spectrometry and theoretical calculations. (a) SERS spectra of pATP from nanoparticles on $\mathrm{Ag}$ and $\mathrm{Au}$ films, which agrees well with theoretical calculations. ${ }^{13}$ (b) Surface mass spectra of pATP absorbed on Ag electrodes, illuminated with laser light (i), without any irradiation (ii), and a roughened Ag-free spectrum of pATP (iii), and the SERS spectra of pATP and DMAB on the Ag electrode. Adapted from ref. 13 and 14.

tion could be attributed to a plasmon catalyzed reaction. ${ }^{6,53}$ In addition to the previously mentioned mass spectroscopy and potential dependent experiments selected plasmon catalysed reactions were also studied at different $\mathrm{pH}$ values, adding a further parameter to control such type of reactions. ${ }^{54-57}$ Some catalytic reactions can be even observed on a single nanoparticle level ${ }^{58,59}$ and single molecule level. ${ }^{26}$

\subsection{Remote plasmonic catalysis in Re-SERS}

Due to the atomic scale roughness and nearly perfect crystal form, chemically synthesized silver and gold nanowires (some micrometer long and some nanometers in diameter) can serve as plasmonic waveguides and play a primary role in remote controlled plasmonic catalysis, which was first reported by Sun et al. in 2011. ${ }^{45}$ As shown in Fig. 5, the remote SERS signals from a junction of silver nanowires and nanoparticles demonstrate that pNTP can react to DMAB induced by remotely excited PSPs. The SEM and optical images of the remote system are shown in Fig. 5(a) and (b). While an incident laser was focused on one of the ends (point A), a tiny emission spot at the junction (point B) and the other end (point C) indicates PSPs efficiently propagated along the Ag nanowire, coupling
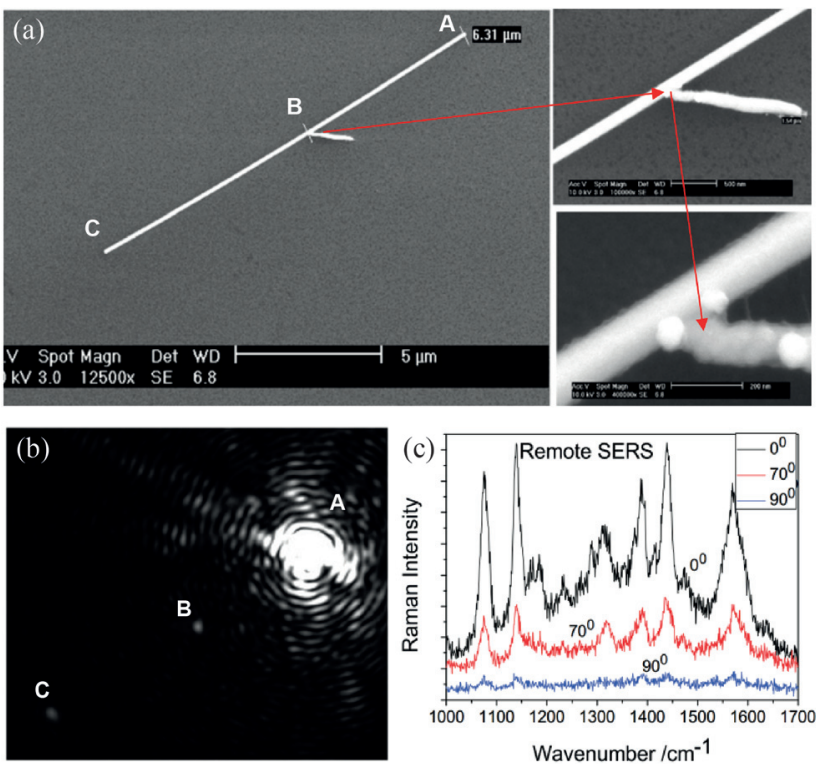

Fig. 5 Remote controlled plasmonic catalysis on a single silver nanowire. (a) SEM images of the remote system of Ag nanowire and nanoparticles, (b) the bright field optical image of PSPs propagating along the Ag nanowire if the incident polarization is parallel to the nanowire axis, (c) polarization angle dependent Re-SERS spectra, which collected at point $B$ in the image of (a). Adapted from ref. 45.

out as photons at point $\mathrm{B}$ with a massive electromagnetic enhancement. Fig. 5(c) shows Re-SERS spectra of the plasmon catalyzed reaction of pNTP to DMAB. The characteristic vibrational modes of DMAB were detected in the Re-SERS spectra, confirming the successful reaction initiation by the PSPs. Since PSPs on metal nanowires depend on the polarization angle of the incident laser, controlled remote plasmonic catalysis can be accomplished by irradiation angle variation. The most effective catalysis was achieved when the incident polarization was parallel to the nanowire axis, which could be understood by the properties of PSPs propagating along the metal nanowires. ${ }^{60}$

In 2012 Lee and Moskovits reported an efficient remote plasmonic sensing using a nanowire array produced by electrochemical deposition on a porous anodic alumina template. ${ }^{11,44}$ This sensing device could be attached to an optical fiber or a catheter to remotely collect SERS signals. The plasmon catalyzed reaction of pATP to DMAB can be realized and monitored by this remote probe, although at that time it was not comprehended that the SERS signals were those of DMAB. ${ }^{44}$ The schematic of the experimental setup and SEM images of the remote sensing structures are shown in Fig. 6 left (a)-(e). For standard SERS measurements the incident laser was directly focused on the top of the wires where the molecule was located. In case of Re-SERS it was focused on the bottom of the glass substrate. In contrast to the above mentioned Re-SERS experiment, the PSPs on the Ag nanowire were excited by the incident laser and by the Raman scattering of the molecules adsorbed on the top of Ag nanowires, so the real 

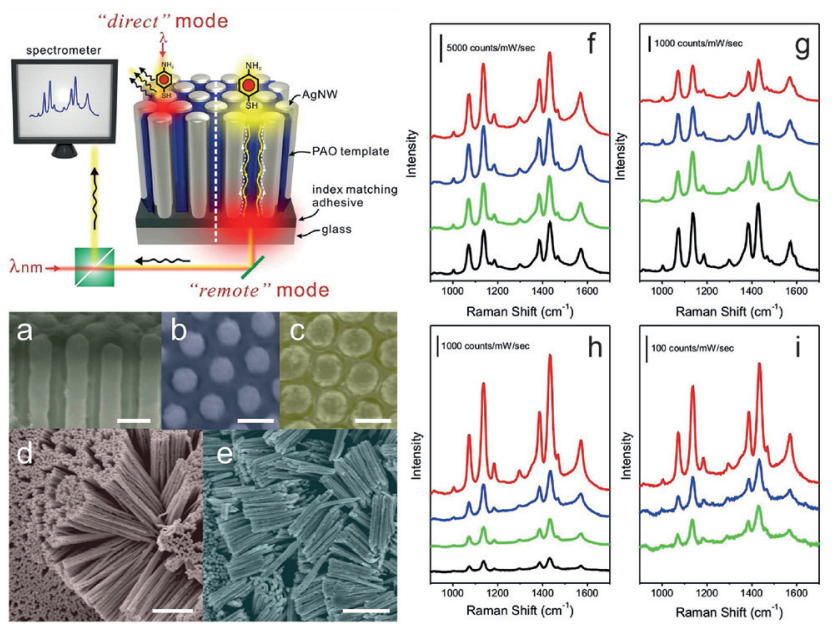

Fig. 6 Remote plasmonic catalysis by a silver nanowire array of pATP to DMAB. Left: scheme of the remote SERS sensor system (top), crosssection (a) and top views (b-e), and the scale bar is $100 \mathrm{~nm}$ in (a)-(c), and $1 \mu \mathrm{m}$ in (d) and (e). Right: averaged SERS spectra by direct excitation ( $f$ and $g$ ) and remote excitation ( $h$ and $i)$; ( $f$ and $g$ ) gap of $11 \mathrm{~nm}$ with nanowire length of $920 \mathrm{~nm}$ (red). Image courtesy of Prof. Moskovits, adapted from ref. 44 .

remote distance was twice the nanowire length. The direct and remote excitation SERS spectra of pATP (DMAB) are shown in the right panel of Fig. 6 . In this work also the influence of the gap distance was investigated, and it turned out that length and diameter of the Ag nanowires influence SERS and Re-SERS signals. The SERS and Re-SERS spectra from a gap of $11 \mathrm{~nm}$ and a length of $0.92-3.3 \mu \mathrm{m}$ are shown in (f) and (g), and the gap of $33 \mathrm{~nm}$ with a length of $0.86-2.88 \mu \mathrm{m}$ are shown in (h) and (i), respectively. It was found that for nanowire arrays with $11 \mathrm{~nm}$ gaps and $3.3 \mu \mathrm{m}$ lengths (i.e., after a $\sim 6.6 \mu \mathrm{m}$ round tip) the Re-SERS signals were rather strong, $\sim 5 \%$ the intensity of those obtained by local SERS.

\section{Plasmonic catalysis in TERS}

\subsection{TERS}

Tip enhanced Raman scattering (TERS) is a technique that provides molecular information on the nanoscale, which was first reported in 2000. ${ }^{61-63}$ In contrast to SERS, TERS can spatially resolve beyond the diffraction limit and is used for single molecule sensing ${ }^{64-69}$ and bio-molecule detection, ${ }^{70-72}$ plasmonic catalysis, ${ }^{73-75}$ and plasmonic gradient effects, ${ }^{76-78}$ to name only a few application areas. In TERS Raman microscopy is combined with scanning probe microscopy (SPM), and an ultra-sharp metal SPM tip can create a hot spot to strongly enhance the Raman scattering intensity. As an example a schematic of a side-illumination TERS setup is shown in Fig. 7. The highly localized enhancement provides a high spatialresolution for optical analysis $(<5 \mathrm{~nm})$, which depends on the nanoparticle nature at the apex of the SPM tip. Commonly SPMs used in TERS include scanning tunnelling microscopes



Fig. 7 Schematic TERS side-illumination setup. A long working distance objective links a Raman system (not shown) and focuses on the plasmonic tip; the enhanced signals originate predominantly from molecules in closest vicinity to the tip apex, which provides the high spatial-resolution analysis. Adapted from ref. 79.

(STM) and atomic force microscopes (AFM). If the tip and substrate were placed in a high vacuum chamber, so called high vacuum TERS (HV-TERS), a clean environment for TERS study is provided which is in particular suitable for studying model plasmonic catalysis. $^{79}$

\subsection{Controlled plasmonic catalysis in TERS}

Plasmon catalyzed reactions revealed by TERS was first reported in 2012. ${ }^{73,74}$ As shown in Fig. 8(a), a monolayer of pNTP was assembled on a smooth gold nanoplate on glass, and probed with a AFM-TERS system. Excitation and collection of the Raman signals were performed through sample support and sample in epi-illumination, and a silver-coated AFM tip was used to generate a strong plasmon intensity to catalyse

(a)

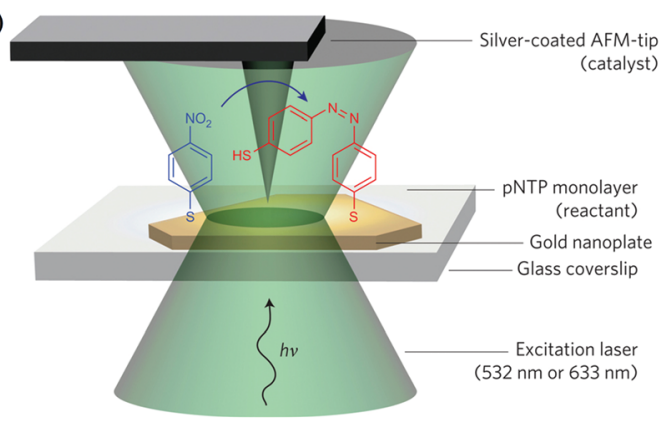

(b)



Fig. 8 Schematic of the plasmon catalyzed reaction of pNTP in TERS. (a) A monolayer pNTP adsorbed on gold nanoplate on glass was catalyzed to DMAB by TERS based on an AFM system. ${ }^{73}$ (b) Same plasmonic catalysis in HV-TERS based on a STM system. ${ }^{74}$ Adapted from ref. 73 and 74. 
pNTP-to-DMAB conversion. The incident laser was switched between green $(532 \mathrm{~nm})$ and red $(632.8 \mathrm{~nm})$ to respectively initiate the reaction (green) and spectroscopically monitor (red). Fig. 8(b) shows the plasmon catalyzed reaction of pNTP to DMAB under high vacuum conditions based on a STM-TERS system. The hot electrons generated from the plasmon decay around the gap between gold tip and silver substrate served as the actual agent. Two pNTP molecules localized in the gap between tip and substrate were catalyzed to a DMAB molecule with the help of hot electrons.

In contrast to the plasmon catalyzed reaction by SERS, an effective control of the catalytic process can be achieved with TERS. Fig. 9(a)-(e) gives incident laser power dependent TERS spectra of the reaction of pNTP to DMAB from a STM-TERS experiment. Initially, the spectrum shows the three main peaks of pNTP (see Fig. 9(a)) at $10 \mu \mathrm{W}$. On increasing the laser intensity to $60 \mu \mathrm{W}$ no spectral changes were observed (see Fig. 9(b)). When the laser intensity was raised to $200 \mu \mathrm{W}$ (see Fig. 9(c)) the intensity of the characteristic band at $1332 \mathrm{~cm}^{-1}$ for the $-\mathrm{NO}_{2}$ stretching mode decreased while DMAB bands at 1140, 1387 and $1432 \mathrm{~cm}^{-1}$ appeared, indicating a gradual reaction. Almost all pNTP molecules dimerized immediately when the laser intensity was increased to $2 \mathrm{~mW}$ (see Fig. 9(d)). Lastly, the spectra did not change when the laser intensity was reduced to the initial $10 \mu \mathrm{W}$ (see Fig. 9(e)). Evidently, a series of in situ spectra in Fig. 9(a) were performed in a long time TERS measurement.

In STM-TERS, a plasmon catalytic reaction cannot only be controlled by the laser intensity, but also by the tip-substrate gap distance. Normally, the decrease of the gap distance can increase the local EM field, and excites a higher surface plasmon intensity at the apex of metallic tip. ${ }^{80}$ Similar to the increase of the incident laser intensity, a faster dimerization of
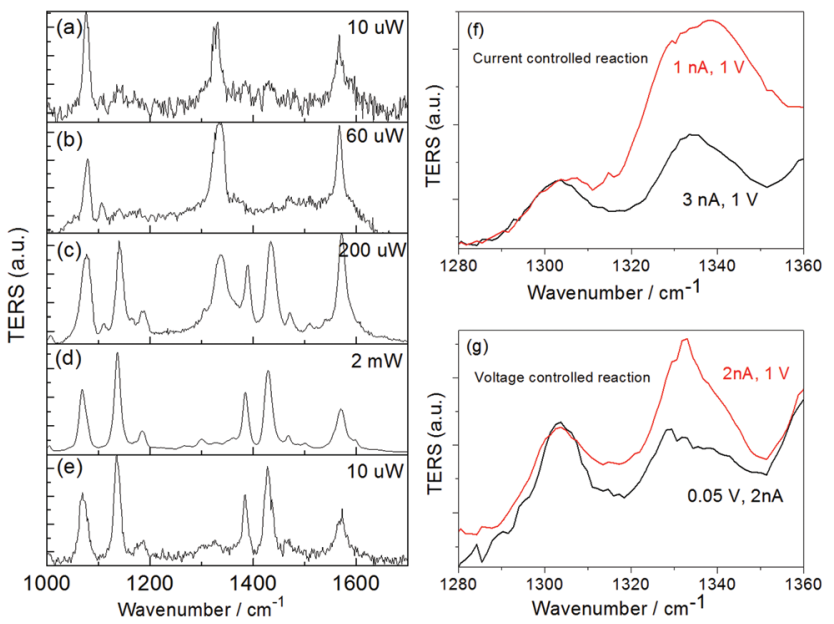

Fig. 9 Laser intensity and gap distance dependent TERS spectra. Laser intensity controlled plasmon catalyzed reaction of pNTP to DMAB at a power of (a) 10, (b) 60, (c) 200, (d) $2000 \mu \mathrm{W}$, and back to (e) $10 \mu \mathrm{W}$; peak of pNTP at $1332 \mathrm{~cm}^{-1}$ can decrease when increasing the tunnelling current ( $f$ ) or decreasing the bias voltage $(g)$. Adapted from ref. 74 .
pNTP to DMAB can be obtained by decreasing the gap distance. The tunnelling current in STM can be written as,

$$
I \propto V \exp \left(\frac{-2 d \sqrt{2 m \Phi}}{\hbar}\right)
$$

where $I$ and $V$ are the tunnelling current and bias voltage, respectively, $d$ is the gap distance between the tip and substrate. According to eqn (1), the gap distance $d$ can be decreased when increasing the tunnelling current $I$ or decreasing the bias voltage $V$. As shown in Fig. 9(f) and (g), an increase of the tunnelling current of 1 to $3 \mathrm{nA}$ at a constant bias voltage of $1 \mathrm{~V}$ results in a decrease of the gap distance and the marker band at $1332 \mathrm{~cm}^{-1}$ decreases, which indicates that more pNTP molecules react to DMAB. Similarly, with a decrease of the bias voltage of $1 \mathrm{~V}$ to $0.05 \mathrm{~V}$ at a constant tunnelling current of $2 \mathrm{nA}$, the peak of pNTP at $1332 \mathrm{~cm}^{-1}$ also decreases. It should be noted that normally chemical bonds could be selectively broken or formed by the tunnelling electron current. Since decreasing the bias voltage at a constant tunnelling current also promotes the plasmon catalyzed reaction, the contribution of the STM tunnelling current to the experiments can be disregarded.

\subsection{Localized temperature of plasmonic catalysis revealed by anti-Stokes TERS}

Due to the high-sensitivity and high spatial-resolution of TERS, the localized temperature can be calculated from the Stokes and anti-Stokes spectra. ${ }^{81,82}$ As shown in Fig. 10(a), the Stokes and anti-Stokes signals from a STM-TERS experiment were observed at a tunnelling current of $2.5 \mathrm{nA}$ and a bias voltage of $1 \mathrm{~V}$. The marker bands of DMAB at 1140, 1387 and $1432 \mathrm{~cm}^{-1}$ can also be clearly observed experimentally in Stokes and anti-Stokes TERS spectra, which further confirmed that almost all pNTP reacted to DMAB.
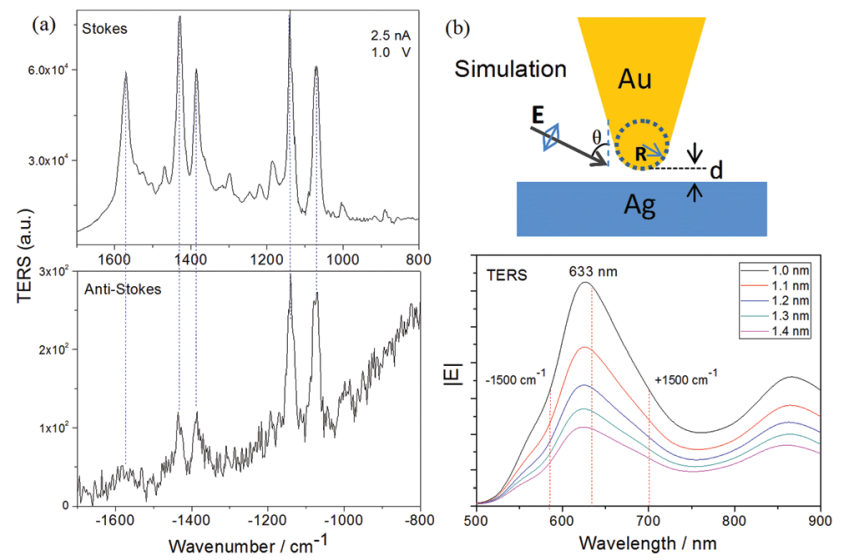

Fig. 10 (a) Stokes and anti-Stokes TERS spectra at a tunnelling current of $2.5 \mathrm{nA}$ and a bias voltage of $1 \mathrm{~V}$, and (b) gap distance dependent simulated spectra of the wavelength scanning plasmon enhancement of TERS, where $R$ is $25 \mathrm{~nm}$ and $\theta=60^{\circ}$. Adapted from ref. 82 . 
The local reaction temperature in plasmonic catalysis can be estimated by fitting the intensities to

$$
I_{\mathrm{S}} / I_{\mathrm{as}}=a \times \mathrm{e}^{\left(h v / k_{\mathrm{B}} T\right)}
$$

where $I_{\mathrm{s}}$ and $I_{\mathrm{as}}$ are the intensities of Stokes and anti-Stokes Raman, and $h, \nu, k_{\mathrm{B}}$ and $T$ are the Planck constant, frequency, Boltzmann constant, experimental temperature, and $a$ is an experimental constant, which can be written as

$$
a=\left(\frac{\sigma\left(\alpha_{\nu}\right)_{\text {Stokes }}}{\sigma\left(\alpha_{\nu}\right)_{\text {anti-Stokes }}}\right)\left(\frac{\nu_{0}-\nu}{\nu_{0}+\nu}\right)^{-4} \sum_{i=1}^{N} \frac{A_{\text {Stokes }}^{2}}{A_{\text {anti-Stokes }}^{2}}
$$

where $\sigma\left(\alpha_{\nu}\right)$ are frequency dependent Stokes and anti-Stokes Raman scattering cross section, respectively, $\nu_{0}$ is the frequency of the laser, $N$ is the number of active molecules, and $A^{2}$ are the enhancement of Stokes and anti-Stokes Raman scattering in TERS. As the TERS intensity is strongly wavelength dependent and since the temperature is fitted by four peaks at different frequencies (wavelengths), the ratio of the enhancement of Stokes and anti-Stokes at different wavelengths is used to correct the fitting equation. The simulated wavelength dependence of the TERS enhancement is shown in Fig. 10(b). A strong plasmon resonance with a broad maximum around $633 \mathrm{~nm}$ (identical to the incident laser wavelength), and almost no position changes can be found when changing the gap distance from 1 to $1.4 \mathrm{~nm}$. Due to the symmetrical distribution of the plasmon enhancement spectra around $633 \mathrm{~nm}$, the method to estimate the localized experimental temperature is valid for $632.8 \mathrm{~nm}$ laser excitation wavelength. The fitted experimental temperature is $330( \pm 10) \mathrm{K}$, and the experimental parameter $a$ in eqn (2) is $2.06( \pm 0.19)$.

\subsection{More plasmon catalyzed reactions in TERS}

In the previous sections the potential of TERS as a tool to investigate and control plasmon catalyzed chemical reactions on the nanoscale has been reviewed. In addition to the plasmonic dimerization of pNTP to DMAB, the plasmonic dissociation of DMAB could also be accomplished by TERS. It has been reported that such products can be controlled by $\mathrm{pH}$ variation. ${ }^{83}$ As shown in Fig. 11(a), the $-\mathrm{N}=\mathrm{N}$ - bond in DMAB can be selectively dissociated by so-called plasmonic scissors, and pATP was produced from the radical via $\mathrm{H}^{+}$attachment under acidic conditions, while pNTP was obtained under alkaline conditions. At low pH, DMAB in the nanogap was excited by $2 \mathrm{~mW}$ laser and TERS spectra were recorded for 60 minutes. As shown in Fig. 11(b) and (c), no significant changes were obtained during this period. However, with a low tunnelling current (increased gap distance) and a weak laser intensity $(20 \mu \mathrm{W})$ resulting in a very low plasmon intensity, a significantly different spectrum was recorded from DMAB. As shown in Fig. 11(d), the $-\mathrm{N}=\mathrm{N}-$ bond of $\mathrm{DMAB}$ was selectively cleaved, and pATP was formed. Fig. 11(f)-(h) shows the TERS spectra at high $\mathrm{pH}$, collected at 0,10 and 60 minutes, respectively. It was found that the characteristic band of pNTP at $1332 \mathrm{~cm}^{-1}$ slowly appears at $2 \mathrm{~mW}$ laser excitation after 10 minutes, which had been obviously increased at 60 minutes (a)
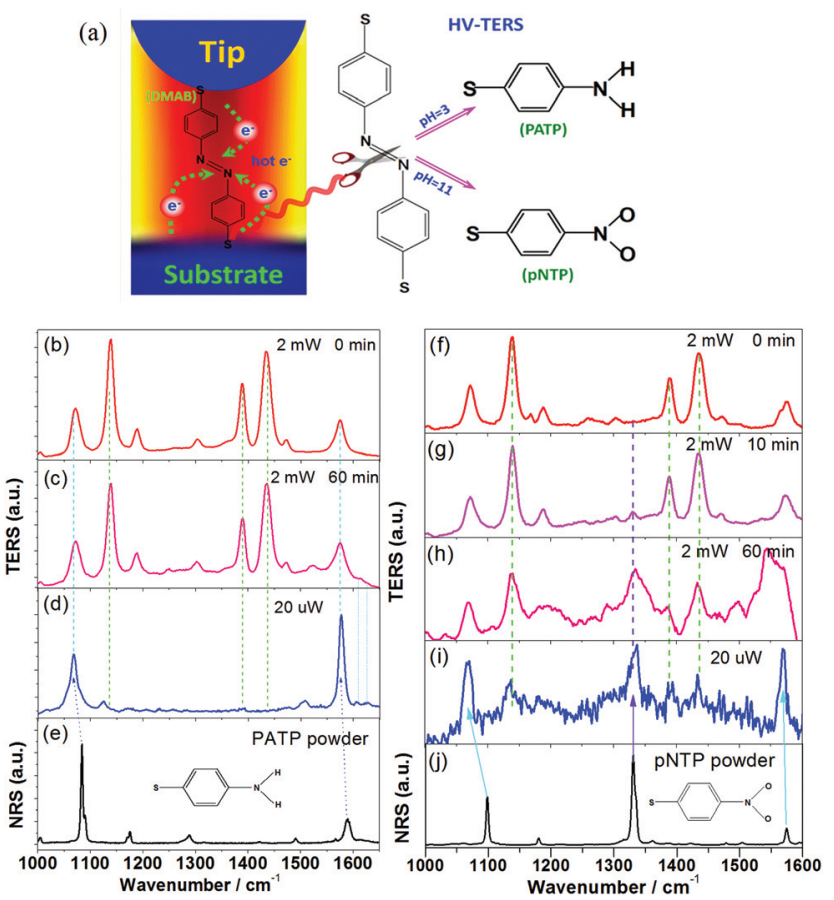

Fig. 11 (a) Schematic of the plasmon catalyzed dissociation of DMAB at low and high $\mathrm{pH}$ values. TERS spectra of DMAB at $\mathrm{pH} 3$ after 0 (b) and 60 (c) minutes, and (d) spectrum on the tip with weak irradiation after strong excitation for 60 minutes. TERS spectra of DMAB at $\mathrm{pH} 11$ after 0 (f), 10 (g) and 60 (h) minutes, and (i) spectrum on the tip with weak irradiation after strong excitation for 60 minutes. Raman spectra of pATP (e) and pNTP (j), respectively. Adapted from ref. 83.

with a simultaneous decrease of the characteristic DMAB signals. The signals of DMAB almost disappeared upon increasing the gap distance and decreasing the laser intensity to $20 \mu \mathrm{W}$, and only the characteristic bands of pNTP were observed (see Fig. 11(i) and (j)). The fragments from DMAB were subsequently converted to pNTP under alkaline conditions.

If the incident laser frequency is close in energy to an electronic transition of molecule, this resonance can enhance the Raman intensity strongly and facilitate a plasmonic catalyzed reaction. Since the absorption maximum of malachite green (MG) is around $630 \mathrm{~nm}$ and the TERS spectrum measured correspondingly at $632.8 \mathrm{~nm}$, it would be expected that an inter or intra molecular reaction of MG could be observed with the help of plasmonic catalysis. This plasmon catalyzed bondselective dissociation of MG molecule with resonant HV-TERS was reported in $2014 .^{75}$ As shown in Fig. 12, resonantly excited Raman bands marked A-D can be observed in spectrum (a). Theoretical calculations reveal that these MG bands are associated with $-\mathrm{NC}_{2} \mathrm{H}_{6}$ fragments (see Fig. 12(f)), whereas band $\mathrm{E}$ refers to a vibrational mode associated with the $\mathrm{C}-\mathrm{C}$ stretching mode of the benzenyl unit. It is noteworthy that the resonance enhanced Raman peaks A-D initially presented in the spectrum (a) disappeared, whereas new peaks $\mathrm{F}-\mathrm{M}$ in the final stable spectrum (c) emerged after 40 minutes. The modes F-M 


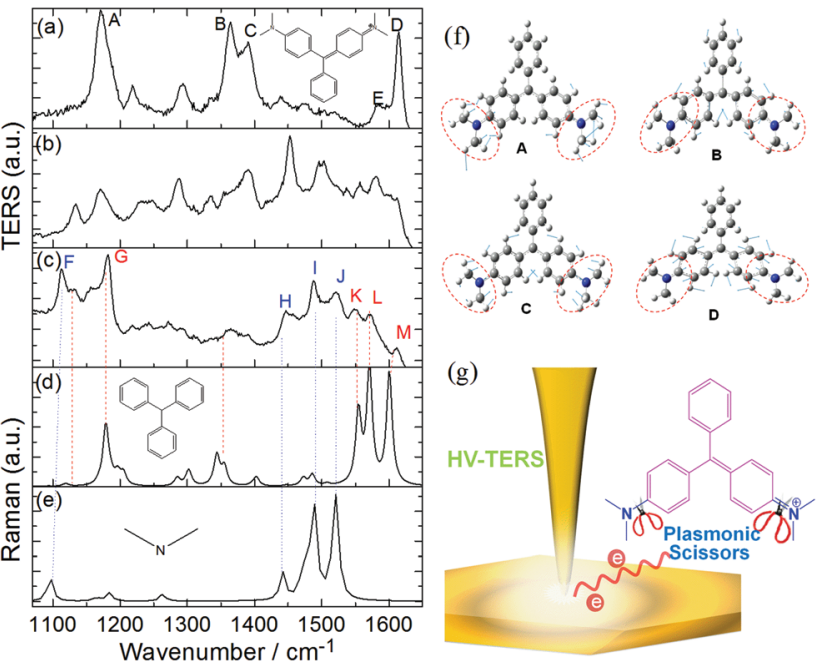

Fig. 12 Time dependent resonant TERS spectra of MG, (a) initial spectrum, (b) intermediate spectrum (20 minutes after continuous laser irradiation), (c) final spectrum (40 minutes after continuous laser irradiation), (d) and (e) simulated Raman spectra of MG fragments. (f) Vibrational modes of MG (corresponding to bands A-D in spectrum (a) as calculated by density functional theory). (g) Sketch of proposed plasmonic scissors in resonant TERS. Adapted from ref. 75 .

can be assigned to the fragments (benzenyl unit of MG) as confirmed by the simulated Raman spectra (d) and (e). It is evident that a bond-selective dissociation reaction must have occurred. Applied to the intramolecular reaction it can be hypothesized that plasmon-induced hot electrons initiate a cleavage of two $-\mathrm{NC}_{2} \mathrm{H}_{6}$ residues from the $\mathrm{MG}$ molecule. A scheme of the proposed plasmonic scissors is shown in Fig. 12(g).

\section{Single molecule level plasmonic catalysis}

As stated above, plasmon catalyzed dimerization of pNTP to DMAB is possible in SERS as well as in TERS. For any dimerization the distance between the reactants is decisive. If the distance between pNTP molecules becomes too large a dimerization reaction cannot occur. In this case the question arises, whether plasmons can initiate an intramolecular reaction such as dissociation or rearrangement. To address this issue, detection sensitivity down to a few or ultimately even a single molecule is required. Using junctions or gaps between nanoparticles, so-called hot spots, very high enhancement factors $\left(10^{8}-10^{12}\right)$ can be achieved, providing sufficient signal enhancement for single molecule detection. ${ }^{84-86}$

A single gold nanoparticle dimer was used for a SERS experiment with pNTP at a potential single molecule level. Different concentrations of the compound were chosen to determine whether it was possible to prevent clustering and self-assembly. Only then the distance of pNTP molecules adsorbed on the nanoparticles becomes too large for an intermolecular reaction. The experiments demonstrated that a dimerization of pNTP to DMAB occurred for $10^{-7}$ and $10^{-8} \mathrm{M}$ solutions of pNTP indicating that the dimer surface was still too densely covered. In $10^{-9} \mathrm{M}$ solution, on average 10 molecules are absorbed on a single gold dimer, resulting in an average distance between two molecules of $\sim 32 \mathrm{~nm}$. As shown in Fig. 13(a), the initial SERS spectrum of pNTP was stable since no DMAB signals could be detected even at laser power within 30 minutes. It was obvious that the distance between pNTP molecules in the hot-spot of the gold dimer was indeed too large to allow an intermolecular reaction. However, an intensity decrease of the strong $1332 \mathrm{~cm}^{-1}$ pNTP signal was observed and after $10 \mathrm{~min}$ the band completely disappeared. Simultaneously new bands at 995 and $1017 \mathrm{~cm}^{-1}$ appeared. Hence, the behavior is considerably different compared to the higher concentrations. The disappearance of the $\mathrm{NO}_{2}$ mode suggests that the entire nitro group was cleaved from the benzene ring resulting in the formation of thiophenol (TP).

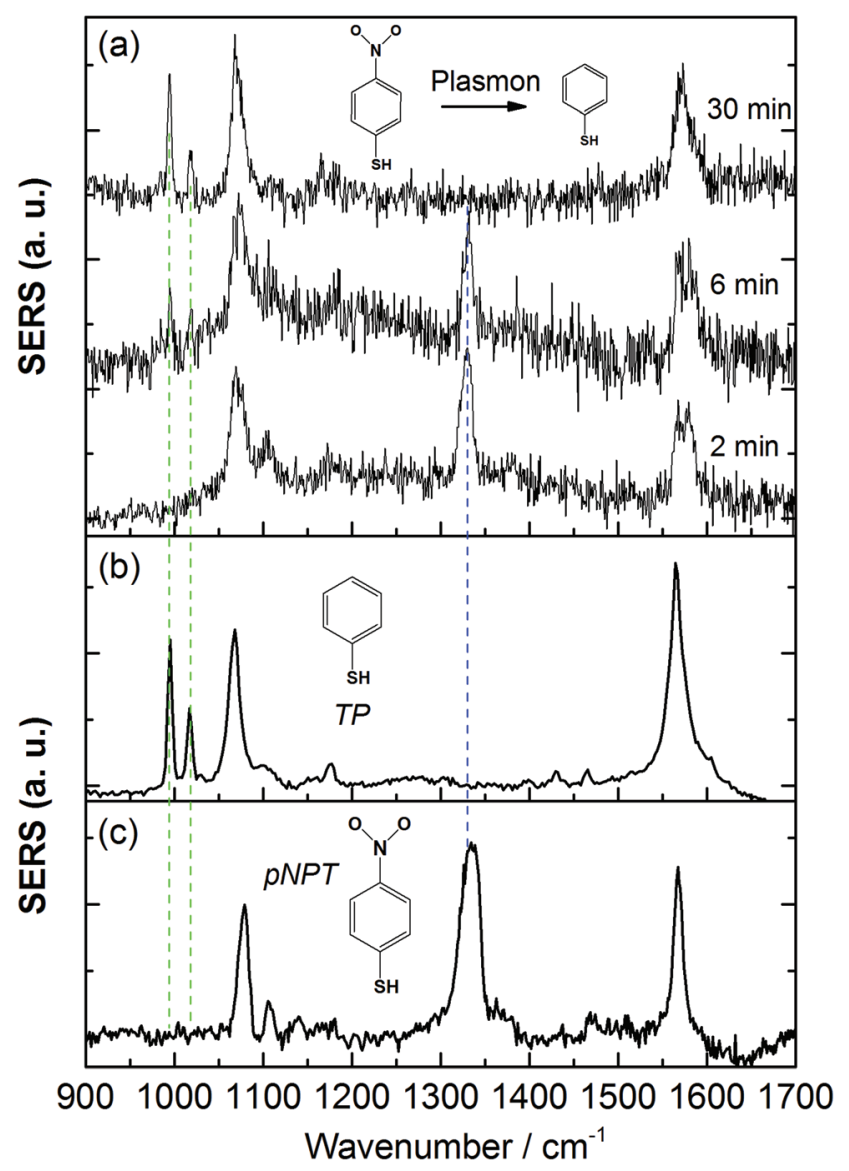

Fig. 13 Comparison of SERS spectra of pNTP and TP with spectra recorded during the experiment by a single gold dimer. (a) Time dependent SERS spectra of reacted pNTP at $3 \mathrm{~mW}$ at $632.8 \mathrm{~nm}$ after 2, 6 and $30 \mathrm{~min}$ irradiation. Normal SERS spectra and corresponding molecular structures of TP and pNTP are shown in (b) and (c), respectively. Adapted from ref. 26. 




Fig. 14 Step-like process of the dissociation of pNTP to TP. Peak intensity is given as a function of time for the pNTP marker band at $1332 \mathrm{~cm}^{-1}$ (red dots), and the TP marker band at $1017 \mathrm{~cm}^{-1}$ (blue triangles). Inset: scheme of the plasmon catalyzed reaction of pNTP to TP near the single molecule level on a single gold dimer.

The striking similarity of SERS spectra of neat TP and those from Fig. 13(b) supports this assumption.

A step-like signal intensity change during the process strongly indicated that the dissociation reaction of pNTP to TP happened close to a single molecule level. In Fig. 14, the peak intensity slope is given as a function of time for the pNTP marker band at $1332 \mathrm{~cm}^{-1}$, and the TP marker band at $1017 \mathrm{~cm}^{-1}$, respectively. Half of the pNTP molecules reacted to TP after $5 \mathrm{~min}$, and both pNTP molecules reacted to TP after $8 \mathrm{~min}$. After $10 \mathrm{~min}$ no significant change occurred. Consequently, it can be assumed that two non-interacting molecules were detected in the hot spot of the single gold dimer. The two pNTP molecules were too far apart from each other for a dimerization reaction and an intramolecular dissociation was initiated.

These experiments clearly support the dimerization mechanism as an explanation for the spectra and also report a novel reaction channel for pNTP, which strongly differs from high concentration investigations.

\section{Summary and outlook}

In this contribution the development of plasmonic catalysis as a new exciting heterogeneous catalytic system on the nanoscale was reviewed. The underlying mechanism was introduced and recent advanced studies of plasmon catalyzed reactions were summarized. In particular pNTP turns out to be a good model/ standard for studies on the nanoscale as SERS, Re-SERS and TERS technique have revealed. With Re-SERS, plasmonic catalysis can be followed and controlled by means of a single Ag nanowire as a waveguide and the reaction is even more efficient with a $\mathrm{Ag}$ nanowire array. In TERS catalytic processes like dimerization, dissociation or protonation can easily monitored by time lapse or laser intensity dependent measurements. Using STM-TERS setups the speed of catalysis can be controlled further by the gap distance between tip and substrate and it was shown that the localized experimental temp- erature can be estimated from the ratio of Stokes and antiStokes TERS spectra. Concentration dependent SERS experiments of pNTP indicate the importance of the concentration of the probed molecule since below a certain threshold the intermolecular reaction switch to an intramolecular process. The ability to follow catalytic reactions near the single molecule level renders enhanced Raman spectroscopy very promising for mechanistic studies. However, to unravel the full potential of plasmonic catalysis, some challenges need to be overcome. In contrast to photocatalysis, the plasmons in plasmonic catalysis play a critical role not only for the excitation process but also for the chemical reaction. Most probably plasmonic catalysis can be attributed to plasmon induced hot electrons, but the detailed mechanism has not been completely understood yet. More theoretical and experimental studies including surface plasmon, hot electrons and plasmonic catalysis are necessary and will certainly follow in the near future to exploit the entire potential of this new research field.

\section{Acknowledgements}

We gratefully acknowledge support from the Deutsche Forschungsgemeinschaft (DEP4TERS, FR1348/19-1) and the Thüringer Aufbau Bank (no. 2011SE9048). Z. Zhang acknowledges financial support from the Alexander von Humboldt foundation.

\section{Notes and references}

1 N. Mizuno and M. Misono, Chem. Rev., 1998, 98, 199-217.

2 A. S. K. Hashmi and G. J. Hutchings, Angew. Chem., Int. Ed., 2006, 45, 7896-7936.

3 A. H. Liang, H. X. Ouyang and Z. L. Jiang, Analyst, 2011, 136, 4514-4519.

4 M. A. Fox and M. T. Dulay, Chem. Rev., 1993, 93, 341-357.

5 J. Schneider, M. Matsuoka, M. Takeuchi, J. L. Zhang, Y. Horiuchi, M. Anpo and D. W. Bahnemann, Chem. Rev., 2014, 114, 9919-9986.

6 M. T. Sun and H. X. Xu, Small, 2012, 8, 2777-2786.

7 K. Ueno and H. Misawa, J. Photochem. Photobiol., C, 2013, 15, 31-52.

8 M. D. Xiao, R. B. Jiang, F. Wang, C. H. Fang, J. F. Wang and J. C. Yu, J. Mater. Chem. A, 2013, 1, 5790-5805.

9 S. Mukherjee, F. Libisch, N. Large, O. Neumann, L. V. Brown, J. Cheng, J. B. Lassiter, E. A. Carter, P. Nordlander and N. J. Halas, Nano Lett., 2013, 13, 240247.

10 S. Mukherjee, L. A. Zhou, A. M. Goodman, N. Large, C. Ayala-Orozco, Y. Zhang, P. Nordlander and N. J. Halas, J. Am. Chem. Soc., 2014, 136, 64-67.

11 J. Lee, S. Mubeen, X. L. Ji, G. D. Stucky and M. Moskovits, Nano Lett., 2012, 12, 5014-5019.

12 H. M. Chen, C. K. Chen, C. J. Chen, L. C. Cheng, P. C. Wu, B. H. Cheng, Y. Z. Ho, M. L. Tseng, Y. Y. Hsu, T. S. Chan, 
J. F. Lee, R. S. Liu and D. P. Tsai, ACS Nano, 2012, 6, 73627372.

13 Y. Fang, Y. Li, H. Xu and M. Sun, Langmuir, 2010, 26, 77377746.

14 Y. F. Huang, H. P. Zhu, G. K. Liu, D. Y. Wu, B. Ren and Z. Q. Tian, J. Am. Chem. Soc., 2010, 132, 9244-9246.

15 W. L. Barnes, A. Dereux and T. W. Ebbesen, Nature, 2003, 424, 824-830.

16 J. Homola, S. S. Yee and G. Gauglitz, Sens. Actuators, B, 1999, 54, 3-15.

17 A. J. Haes and R. P. Van Duyne, Anal. Bioanal. Chem., 2004, 379, 920-930.

18 K. A. Willets and R. P. Van Duyne, Annu. Rev. Phys. Chem., 2007, 58, 267-297.

19 A. Campion and P. Kambhampati, Chem. Soc. Rev., 1998, 27, 241-250.

20 E. Bailo and V. Deckert, Chem. Soc. Rev., 2008, 37, 921-930.

21 H. Wei, D. Ratchford, X. Q. Li, H. X. Xu and C. K. Shih, Nano Lett., 2009, 9, 4168-4171.

22 Y. R. Fang, H. Wei, F. Hao, P. Nordlander and H. X. Xu, Nano Lett., 2009, 9, 2049-2053.

23 A. O. Govorov, H. Zhang and Y. K. Gun'ko, J. Phys. Chem. C, 2013, 117, 16616-16631.

24 I. Goykhman, B. Desiatov, J. Khurgin, J. Shappir and U. Levy, Nano Lett., 2011, 11, 2219-2224.

25 M. W. Knight, H. Sobhani, P. Nordlander and N. J. Halas, Science, 2011, 332, 702-704.

26 Z. L. Zhang, T. Deckert-Gaudig, P. Singh and V. Deckert, Chem. Commun., 2015, 51, 3069-3072.

27 P. L. Stiles, J. A. Dieringer, N. C. Shah and R. R. Van Duyne, Annu. Rev. Anal. Chem., 2008, 1, 601-626.

28 J. A. Dougan and K. Faulds, Analyst, 2012, 137, 545-554.

29 M. Moskovits, J. Raman Spectrosc., 2005, 36, 485-496.

30 M. Fleischm, P. J. Hendra and A. Mcquilla, Chem. Phys. Lett., 1974, 26, 163-166.

31 D. L. Jeanmaire and R. P. Vanduyne, J. Electroanal. Chem., 1977, 84, 1-20.

32 C. L. Haynes and R. P. Van Duyne, J. Phys. Chem. B, 2003, 107, 7426-7433.

33 J. F. Masson, M. P. Murray-Methot and L. S. Live, Analyst, 2010, 135, 1483-1489.

34 Z. L. Zhang, P. F. Yang, H. X. Xu and H. R. Zheng, J. Appl. Phys., 2013, 113, 033102.

35 R. M. Dickson and L. A. Lyon, J. Phys. Chem. B, 2000, 104, 6095-6098.

36 S. Lal, J. H. Hafner, N. J. Halas, S. Link and P. Nordlander, Acc. Chem. Res., 2012, 45, 1887-1895.

37 H. Ditlbacher, A. Hohenau, D. Wagner, U. Kreibig, M. Rogers, F. Hofer, F. R. Aussenegg and J. R. Krenn, Phys. Rev. Lett., 2005, 95.

38 J. A. Hutchison, S. P. Centeno, H. Odaka, H. Fukumura, J. Hofkens and H. Uji-i, Nano Lett., 2009, 9, 995-1001.

39 Y. Z. Huang, Y. R. Fang, Z. L. Zhang, L. Zhu and M. T. Sun, Light: Sci. Appl., 2014, 3.

40 J. R. Xia, Y. T. Feng, L. Zhu, Z. L. Zhang, L. X. Xia and L. W. Liu, Plasmonics, 2014, 9, 673-676.
41 M. T. Sun, Z. L. Zhang, P. J. Wang, Q. Li, F. C. Ma and H. X. Xu, Light: Sci. Appl., 2013, 2, e68.

42 R. X. Yan, J. H. Park, Y. Choi, C. J. Heo, S. M. Yang, L. P. Lee and P. D. Yang, Nat. Nanotechnol., 2012, 7, 191196.

43 Y. Z. Huang, Y. R. Fang and M. T. Sun, J. Phys. Chem. C, 2011, 115, 3558-3561.

44 S. J. Lee and M. Moskovits, J. Am. Chem. Soc., 2012, 134, 11384-11387.

45 M. T. Sun, Y. X. Hou, Z. P. Li, L. W. Liu and H. X. Xu, Plasmonics, 2011, 6, 681-687.

46 P. Song, Y. Z. Li, Y. Q. Li, M. Y. Zhao, L. W. Liu and M. T. Sun, Plasmonics, 2013, 8, 249-254.

47 B. Dong, Y. R. Fang, X. W. Chen, H. X. Xu and M. T. Sun, Langmuir, 2011, 27, 10677-10682.

48 V. Canpean, M. Iosin and S. Astilean, Chem. Phys. Lett., 2010, 500, 277-282.

49 M. Osawa, N. Matsuda, K. Yoshii and I. Uchida, J. Phys. Chem., 1994, 98, 12702-12707.

50 Q. Zhou, X. W. Li, Q. Fan, X. X. Zhang and J. W. Zheng, Angew. Chem., Int. Ed., 2006, 45, 3970-3973.

51 D. Maniu, V. Chis, M. Baia, F. Toderas and S. Astilean, J. Optoelectron. Adv. Mater, 2007, 9, 733-736.

52 A. P. Richter, J. R. Lombardi and B. Zhao, J. Phys. Chem. C, 2010, 114, 1610-1614.

53 L. B. Zhao, M. Zhang, Y. F. Huang, C. T. Williams, D. Y. Wu, B. Ren and Z. Q. Tian, J. Phys. Chem. Lett., 2014, 5, 1259-1266.

54 M. T. Sun, Y. Z. Huang, L. X. Xia, X. W. Chen and H. X. Xu, J. Phys. Chem. C, 2011, 115, 9629-9636.

55 Y. Zong, Q. H. Guo, M. M. Xu, Y. X. Yuan, R. N. Gu and J. L. Yao, RSC Adv., 2014, 4, 31810-31816.

56 P. Singh and V. Deckert, Chem. Commun., 2014, 50, 1120411207.

57 W. Xie, C. Herrmann, K. Kompe, M. Haase and S. Schlucker, J. Am. Chem. Soc., 2011, 133, 19302-19305.

58 L. L. Kang, P. Xu, B. Zhang, H. H. Tsai, X. J. Han and H. L. Wang, Chem. Commun., 2013, 49, 3389-3391.

59 W. Xie, B. Walkenfort and S. Schlucker, J. Am. Chem. Soc., 2013, 135, 1657-1660.

60 S. P. Zhang, H. Wei, K. Bao, U. Hakanson, N. J. Halas, P. Nordlander and H. X. Xu, Phys. Rev. Lett., 2011, 107, 096801.

61 R. M. Stockle, Y. D. Suh, V. Deckert and R. Zenobi, Chem. Phys. Lett., 2000, 318, 131-136.

62 M. S. Anderson, Appl. Phys. Lett., 2000, 76, 3130-3132.

63 N. Hayazawa, Y. Inouye, Z. Sekkat and S. Kawata, Opt. Commun., 2000, 183, 333-336.

64 Z. Liu, S. Y. Ding, Z. B. Chen, X. Wang, J. H. Tian, J. R. Anema, X. S. Zhou, D. Y. Wu, B. W. Mao, X. Xu, B. Ren and Z. Q. Tian, Nat. Commun., 2011, 2, 305.

65 M. D. Sonntag, D. Chulhai, T. Seideman, L. Jensen and R. P. Van Duyne, J. Am. Chem. Soc., 2013, 135, 1718717192.

66 T. Deckert-Gaudig, E. Kammer and V. Deckert, J. Biophotonics, 2012, 5, 215-219. 
67 R. Zhang, Y. Zhang, Z. C. Dong, S. Jiang, C. Zhang, L. G. Chen, L. Zhang, Y. Liao, J. Aizpurua, Y. Luo, J. L. Yang and J. G. Hou, Nature, 2013, 498, 82-86.

68 W. H. Zhang, B. S. Yeo, T. Schmid and R. Zenobi, J. Phys. Chem. C, 2007, 111, 1733-1738.

69 B. Pettinger, Mol. Phys., 2010, 108, 2039-2059.

70 A. Rasmussen and V. Deckert, J. Raman Spectrosc., 2006, 37, 311-317.

71 T. Deckert-Gaudig and V. Deckert, Phys. Chem. Chem. Phys., 2010, 12, 12040-12049.

72 R. Treffer, R. Bohme, T. Deckert-Gaudig, K. Lau, S. Tiede, X. M. Lin and V. Deckert, Biochem. Soc. Trans., 2012, 40, 609-614.

73 E. M. van Schrojenstein Lantman, T. Deckert-Gaudig, A. J. G. Mank, V. Deckert and B. M. Weckhuysen, Nat. Nanotechnol., 2012, 7, 583-586.

74 M. T. Sun, Z. L. Zhang, H. R. Zheng and H. X. Xu, Sci. Rep., 2012, 2.

75 Z. L. Zhang, S. X. Sheng, H. R. Zheng, H. X. Xu and M. T. Sun, Nanoscale, 2014, 6, 4903-4908.

76 M. T. Sun, Z. L. Zhang, P. J. Wang, Q. Li, F. C. Ma and H. X. Xu, Light: Sci. Appl., 2013, 2.
77 M. T. Sun, Z. L. Zhang, L. Chen, S. X. Sheng and H. X. Xu, Adv. Opt. Mater., 2014, 2, 74-80.

78 M. T. Sun, Y. R. Fang, Z. Y. Zhang and H. X. Xu, Phys. Rev. E: Stat. Phys., Plasmas, Fluids, Relat. Interdiscip. Top., 2013, 87.

79 Z. L. Zhang, L. Chen, S. X. Sheng, M. T. Sun, H. R. Zheng, K. Q. Chen and H. X. Xu, Front. Phys, 2014, 9, 17-24.

80 M. T. Sun, Y. R. Fang, Z. L. Yang and H. X. Xu, Phys. Chem. Chem. Phys., 2009, 11, 9412-9419.

81 Z. L. Zhang, X. R. Tian, H. R. Zheng, H. X. Xu and M. T. Sun, Plasmonics, 2013, 8, 523-527.

82 Z. L. Zhang, L. Chen, M. T. Sun, P. P. Ruan, H. R. Zheng and H. X. Xu, Nanoscale, 2013, 5, 3249-3252.

83 M. T. Sun, Z. L. Zhang, Z. H. Kim, H. R. Zheng and H. X. Xu, Chem. - Eur. J., 2013, 19, 14958-14962.

84 S. M. Nie and S. R. Emery, Science, 1997, 275, 1102-1106.

85 K. Kneipp, Y. Wang, H. Kneipp, L. T. Perelman, I. Itzkan, R. Dasari and M. S. Feld, Phys. Rev. Lett., 1997, 78, 16671670.

86 H. X. Xu, E. J. Bjerneld, M. Kall and L. Borjesson, Phys. Rev. Lett., 1999, 83, 4357-4360. 